\title{
Little difference between triclosan and stannous fluoride dentifrices on gingival inflammation
}

\author{
Abstracted from \\ Sälzer S, Slot DE, Dörfer CE, Van der Weijden GA. \\ Comparison of triclosan and stannous fluoride dentifrices on parameters of gingival \\ inflammation and plaque scores: a systematic review and meta-analysis. \\ Int J Dent Hyg 2015; 13: 1-17 doi: 10.1111/idh.12072. [Epub ahead of print] PubMed PMID: 24945592. \\ Address for correspondence: Sonja Sälzer, Department of Periodontology, Clinic for Conservative Dentistry \\ and Periodontology, School for Dental Medicine, Christian-Albrechts-University, Kiel, Arnold-Heller-Straße 3, \\ Haus 26 Kiel 24105, Germany. E-mail: saelzer@konspar.uni-kiel.de
}

\section{Question: Are dentifrices containing triclosan (TcS) more effective than stannous fluoride $(\mathrm{SnF})$ dentifrices on the parameters of gingival inflammation?}

Data sources Medline, the Cochrane Central Register of Controlled Trials and Embase.

Study selection Randomised, controlled trials (RCTs) or controlled clinical trials (CCTs) in adults $\geq 17$ years of age involving either triclosan or stannous-fluoride-containing dentifrices and self-performed manual brushing with a minimum four weeks of follow-up were considered. Data extraction and synthesis Study quality was assessed by two reviewers and meta-analysis performed where using fixed or random effects models as appropriate.

Results Fifteen RCTs including four unpublished trials from one manufacturer met the criteria.

No differences in the gingival index were seen between the two dentifrices [DiffM-0.04, 95\% confidence interval $\mathrm{Cl}(-0.11 ; 0.04)$; $P=0.34]$. However the change in the average gingival bleeding score was significantly in favour of $\mathrm{SnF}$ [DiffM0.02, $95 \% \mathrm{Cl}(0.01 ; 0.02)$; $\mathrm{P}<0.00001]$. Plaque scores demonstrated a statistically significant difference in favour of TCS, according to the Quigley-Hein Plaque Index (Q\&H Pl; DiffM-0.29, 95\% Cl [-0.45; -0.13]; P = 0.0004), but there was no difference according to Rustogi Modified Navy Plaque Index (RMNPI) [DiffM-0.09, 95\% Cl $(-0.01 ; 0.18) ; \mathrm{P}=0.07$ ].

Conclusions In the context of inconclusive results for the primary outcome variable of gingival health, it can be concluded that there was a minor and most likely clinically insignificant difference between Tcsand SnF-containing dentifrices. Meta-analysis of plaque score reduction was also inconclusive; whereas TCS was more effective when assessed by the Q\&H PI, it was not when scored with the RMNPI.

\section{Commentary}

The systematic review by Salzer et al. ${ }^{1}$ addressed a focused clinical question 'What are the effects of triclosan-containing dentifrices compared with SnF-containing dentifrices on the parameters of gingivitis and on plaque scores in healthy subjects aged at least 17 years?' The quality of this systematic review is good as assessed by AMSTAR ('assessment of multiple systematic reviews') measurement tool. ${ }^{2}$ Previous reviews ${ }^{3-5}$ have shown that both triclosan (Tcs) and stannous fluoride $(\mathrm{SnF})$ are effective in plaque reduction and gingivitis when compared to other fluoride-containing dentifrices. However, there has been no systematic review that looked at direct comparisons between Tcs-containing and SnF-containing dentifrices. The authors of this review set to perform the same.

They followed a very thorough process in conducting this systematic review by identifying all randomised and controlled clinical studies done testing the efficacy of Tcs-containing and SnFcontaining dentifrices. They pursued additional steps to obtain unpublished data from the manufacturers of the dentifrices which are usually rare in the majority of systematic reviews. The authors of this review finalised a total of 15 studies (11 published and four unpublished from Procter \& Gamble Co.) to address the focused question. All the included studies were found to have considerable heterogeneity in many important areas including the study design, study duration, clinical indices used and different toothbrushes among others.

All the studies were assessed to have moderate risk of bias with possible publication bias. The outcome measures of gingival index and plaque scores were found to be inconsistent for Tcs- and SnFcontaining dentifrices between the studies. Only the bleeding score outcome was considered to have consistent results, but the reader needs to remember that the included studies had moderate risk of bias as well as publication bias. It was very interesting to note that the authors of this review found that the unpublished and non-sponsored studies did not favour either Tcs or SnF products. The adverse effect mainly discussed was tooth staining in some studies for Tcs dentifrices, which was however not supported in other studies.

The authors of this review based on their thorough quality and statistical assessment fairly conclude that there is only minor and most likely clinically insignificant difference between Tcs-containing and SnF-containing dentifrices on the gingival outcome measures and hence the strength of recommendation for either of these dentifrices is weak. Lastly, the authors of this review found that the included studies did not report important 


\section{PERIODONTAL}

trial information such as allocation concealment and sample size determination. Authors of randomised controlled studies should follow the recommended guidelines by CONSORT which will eliminate bias to a great degree while assessing the clinical usefulness of these studies. ${ }^{6}$

\section{Practice point}

- Inconclusive and weak evidence show minor but clinically insignificant difference between triclosan- and stannous fluoridecontaining dentifrices in gingival health parameters.
Satish Kumar

Division of Periodontology, Diagnostic Sciences \& Dental Hygiene, Ostrow School of Dentistry of USC, Los Angeles, CA, USA

1. Sälzer S, Slot DE, Dörfer CE, Van der Weijden GA. Comparison of triclosan and stannous fluoride dentifrices on parameters of gingival inflammation and plaque scores: a systematic review and meta-analysis. Int J Dent Hyg 2015; 13: 1-17.

2. Shea BJ, Grimshaw JM, Wells GA, et al. Development of AMSTAR: a measurement tool to assess the methodological quality of systematic reviews. BMC Med Res Methodol 2007; 7: 10 .

3. Hioe KP, van der Weijden GA. The effectiveness of self-performed mechanical plaque control with triclosan containing dentifrices. Int J Dent Hyg 2005; 3: 192-204.

4. Paraskevas $S$, van der Weijden GA. A review of the effects of stannous fluoride on gingivitis. J Clin Periodontol 2006; 33: 1-13.

5. Niederman R. Stannous fluoride toothpastes reduce the gingival index more than sodium fluoride toothpastes. Evid Based Dent 2007; 8: 74-75.

6. Schulz KF, Altman DG, Moher D; CONSORT Group. CONSORT 2010 statement: updated guidelines for reporting parallel group randomised trials. Br Med J 2010; 340: $\mathrm{c} 332$.

Evidence-Based Dentistry (2015) 16, 13-14. doi:10.1038/sj.ebd.6401076 